\title{
Saúde mental em comunidades quilombolas do Brasil: uma revisão sistemática da literatura
}

\author{
Mental health in Brazil's quilombola communities: \\ a systematic review of the literature \\ Salud mental en comunidades quilombolas de Brasil: \\ una revisión sistemática de la literatura
}

\author{
Eraldo Carlos Batista ${ }^{1}$ \\ Katia Bones Rocha ${ }^{1}$
}

Recebido em 10/09/2018; revisado e aprovado em 29/04/2019; aceito em 06/05/2019.

DOI: http://dx.doi.org/10.20435/inter.v21i1.2149

\begin{abstract}
Resumo: O objetivo desta revisão foi identificar estudos sobre saúde mental desenvolvidos nas populações quilombolas. Os artigos foram pesquisados em três bancos de dados: Lilacs, Medline e SciELO, utilizando os seguintes descritores: "quilombola OR quilombo AND mental health OR mental disorders". Foram selecionados oito estudos que atenderam aos critérios de inclusão. A maioria dos trabalhos apontou os indicadores sociais e econômicos como fatores associados à condição de saúde e saúde mental dos membros das comunidades quilombolas.
\end{abstract}

Palavras-chave: saúde mental; saúde pública; determinantes sociais de saúde.

Abstract: This review's objective was to verify studies on mental health developed with quilombola populations. The researches were taken from three different databases: Lilacs, Medline and SciELO, using the following describers: "quilombola OR quilombo AND mental health OR mental disorders". We selected eight studies, which met the inclusion criteria. Most of the studies pointed social and economic indicators as factors associated to the health and mental health conditions of members of quilombola communities. Keywords: mental health; public health; social determinants of health.

Resumen: El objetivo de esta revisión fue identificar estudios sobre salud mental desarrollados en poblaciones quilombolas. Los artículos fueron investigados en tres bancos de datos: Lilacs, Medline y SciELO, utilizando los siguientes descriptores: "quilombola OR quilombo AND mental health OR mental disorders". Fueron seleccionados ocho estudios que atendieron los criterios de inclusión. La mayor parte de los trabajos apuntó los indicadores sociales y económicos como factores asociados a la condición de salud y salud mental de los miembros de las comunidades quilombolas.

Palabras clave: salud mental; salud pública; determinantes sociales de salud.

\section{INTRODUÇÃO}

As comunidades quilombolas sofrem com o forte impacto da desigualdade social. Por estarem localizadas principalmente nas áreas rurais, longe dos centros urbanos, essas comunidades vivem num relativo grau de isolamento geográfico, em locais de difícil acesso e de desassistência. Além disso, o preconceito e a discriminação sofridos por esse grupo agravam as situações de descaso e negligência do poder público, especialmente no que diz respeito ao acesso à saúde de qualidade (XAVIER, 2012; MONEGO et al., 2010).

Apesar dos avanços, como a certificação por parte da Fundação Cultural Palmares, as populações remanescentes de quilombos ainda sofrem uma diversidade de problemas. À medida que as comunidades quilombolas passaram a contar com um reconhecimento oficial de identidade e cultura, passaram a sofrer consequências diretas na manutenção de sua sobrevivência. De acordo com o Observatório Quilombola, ações externas indiscriminadas caracterizadas por

\footnotetext{
${ }^{1}$ Pontifícia Universidade Católica do Rio Grande do Sul (PUCRS), Porto Alegre, Rio Grande do Sul, Brasil.
} 
interferências em âmbitos político-sociais, ambientais, educativos, culturais e de saúde têm, notoriamente, fragilizado as comunidades quilombolas no Brasil. Essas ações resultam em ameaça à identidade, à autonomia, ao modo de organização do trabalho e às condições de permanência em seus locais de origem e, consequentemente, à saúde (VAREJÃO et al., 2006).

Estudos, sob diversos enfoques, em comunidades quilombolas de várias regiões do Brasil têm apontado para a necessidade de efetivação de ações de políticas públicas voltadas à população negra (BEZERRA et al., 2014; MARQUES et al., 2014; MELO; SILVA, 2015; MONEGO et al., 2010). Esses estudos corroboram dados oficiais do Governo, os quais apontam que a população afrodescendente, juntamente dos indígenas, continua sendo uma das mais desfavorecidas, com níveis mais altos de ruralidade, pobreza, desemprego, analfabetismo e migração, associados a menor acesso aos serviços de saúde e saneamento ambiental (BRASIL, 2016), refletindo a vulnerabilidade social em que vivem. No que se refere às condições de saúde nas comunidades quilombolas, a desigualdade social tem efeitos diretos no acesso aos cuidados e no agravo dos problemas de saúde desse grupo.

Outras pesquisas sugerem a associação entre problemas básicos de saúde das comunidades quilombolas e precárias condições de vida e moradia, ausência de saneamento básico e acesso restrito à educação dessas comunidades (BEZERRA et al., 2014; CAVALCANTE, 2011; FREITAS et al., 2011; OLIVEIRA et al., 2012). A investigação desses marcadores sociais e suas relações com a saúde permite a compreensão do processo saúde-doença nas comunidades quilombolas, não apenas em seus aspectos biológicos, mas também considerando os determinantes sociais da saúde (SOUZA; SILVA; SILVA, 2013).

Os determinantes sociais da saúde correspondem às condições de vida, trabalho e moradia dos indivíduos e comunidades, associados aos fatores sociais, econômicos, culturais, étnico/raciais, psicológicos e comportamentais que influenciam no processo de saúde e doença (KRIEGER, 2001; FREITAS et al., 2011; BUSS; PELLEGRINI FILHO, 2007). No entanto Krieger (2001) afirma que todos esses fatores que produzem as desigualdades em saúde são evitáveis e poderiam ser modificados com ações em diversos níveis.

De forma mais específica, no campo da saúde mental, os determinantes sociais de uma comunidade são particularmente relevantes na compreensão e na construção de novos modos de estar diante da pessoa em adoecimento mental (BOSI et al., 2014). Dimenstein et al. (2017), seguindo o proposto pelo Centro Brasileiro de Estudos em Saúde (Cebes) e pela Associação Latino-Americana de Medicina Social (Alames), propõem uma substituição da expressão "determinantes" por "determinação social", uma vez que a primeira está relacionada a uma visão linear e que pode ser reducionista em relação ao processo saúde-doença. Desse modo, a noção de determinação social é entendida como forma de apreensão das múltiplas determinações que incidem em uma realidade concreta, destacando os aspectos relacionais e do cotidiano de vida nas comunidades (DIMENSTEIN et al., 2017). Contudo, neste estudo, a utilização do termo "determinantes" se insere em uma compreensão não linear do processo saúde/doença.

Ao estudar a relação entre os marcadores sociais e sua relação com a saúde mental, foi encontrada associação da maior prevalência de problemas de saúde mental entre pessoas de classe social menos privilegiada, com menores níveis de estudo, imigrantes, com um maior número de doenças crônicas e que referiram pouco apoio social (ROCHA et al., 2015). Nessa mesma direção, a Organização Mundial de Saúde (OMS) destaca que a distribuição da prevalência de transtornos mentais não ocorre de forma igualitária em toda a população (OMS, 2001). Ou 
seja, grupos que se encontram em desvantagens decorrentes de relações e interações sociais desiguais, como a população negra, que traz consigo um conjunto de variáveis individuais e contextuais como componentes ligados ao racismo (iniquidades, segregação e exclusão étnica) e condições socioeconômicas desfavoráveis, podem se tornar mais propensos a desenvolver transtornos mentais, como apontam alguns indicadores de utilização de serviços de saúde a respeito de que as comunidades negras estão sempre em desvantagem quando comparadas à população branca (BRASIL, 2016).

Desse modo, o conhecimento dos determinantes sociais e das variáveis que produzem desigualdades em saúde mental e sua influência no adoecimento da população quilombola ganham relevância, à medida que esses elementos são integrados na formulação de políticas que orientam as prioridades para a gestão pública em saúde para com essa população (ALVES; RODRIGUES, 2010; FERREIRA; LATORRE, 2012). Porém Gama, Campos e Ferrer (2014) alertam que as relações entre saúde-doença mental e vulnerabilidade social são muito complexas e que sua compreensão exige reflexões e contextualizações que não reforcem o preconceito e a estigmatização em relação a grupos de classe social menos privilegiada, por meio de associação entre sofrimento mental e pobreza. Entretanto a problematização dessas relações pode sinalizar novos caminhos e boas práticas na área de saúde mental.

Nessa compreensão, a saúde-doença mental deve ser pensada como o produto de múltiplas e complexas interações, que incluem fatores biológicos, psicológicos e sociais (ALVES; RODRIGUES, 2010), considerando o conceito de saúde mental de forma mais ampla, de maneira que este possa refletir a conjuntura social, econômica, política e cultural da sociedade, sem representar a mesma coisa para todas as pessoas. De outra maneira, a definição de saúde-doença mental dependerá da época, do lugar, da classe social, de valores individuais, de concepções científicas, religiosas e filosóficas (FREITAS et al., 2011).

Nessa direção, a Portaria n. 992, de 13 de maio de 2009, que instituiu a Política Nacional de Saúde Integral da População Negra (PNSIPN), tem como objetivo promover a saúde integral da população negra, priorizando a redução das desigualdades étnico-raciais, assim como o combate ao racismo e à discriminação nas instituições e serviços do SUS. Em seu Capítulo III, inciso V, a PNSIPN propõe o fortalecimento da atenção à saúde mental das crianças, adolescentes, jovens, adultos e idosos negros, com vistas à qualificação da atenção para o acompanhamento do crescimento, desenvolvimento e envelhecimento e a prevenção dos agravos decorrentes dos efeitos da discriminação racial e exclusão social. Ainda no mesmo Capítulo, o inciso VI enfatiza o fortalecimento da atenção à saúde mental de mulheres e homens negros, em especial aqueles com transtornos decorrentes do uso de álcool e outras drogas (BRASIL, 2009).

Diante do que foi referido e considerando a escassez de estudos que investigam os fatores individuais e contextuais associados ao sofrimento mental em populações rurais (DIMENTEIN et al., 2017) e, mais especificamente, em populações quilombolas no Brasil (BARROSO; MELO; GUIMARÃES, 2015), entende-se que a construção de novos saberes no campo da saúde mental dessa população requer a compreensão dos seus determinantes sociais que se materializam em elementos produtores de desigualdades.

Desse modo, este estudo teve o objetivo de identificar, mediante uma revisão sistemática, estudos sobre saúde mental desenvolvidos nas populações quilombolas. Outro objetivo nesta revisão foi analisar se esses estudos consideram os determinantes sociais da saúde para compreender o processo de saúde e doença dessa população. 


\section{MÉTODO}

O presente estudo inspirou-se nas recomendações da Preferred Reporting Items for Systematic Reviews and Meta-Analyses (PRISMA), que visam orientar a elaboração de revisões sistemáticas da literatura e meta-análises na área da saúde humana (MOHER et al., 2009). O primeiro passo foi formular a questão da pesquisa, que consistiu em identificar quais são os fatores associados ao sofrimento mental em membros de comunidades remanescentes quilombolas no Brasil e como os determinantes sociais da saúde aparecem ou não nessa discussão do processo de saúde e doença mental.

No processo de construção dos descritores, foram realizados diversos testes com diferentes descritores a fim de encontrar um que contemplasse o maior número de estudos sobre o assunto. Depois de realizados os testes, foi utilizada a seguinte combinação no SciELO e no Lilacs: (saúde mental or problemas de saúde mental or transtorno mental or depressão) AND (quilombola or quilombo); na Medline: (saúde mental) $O R$ (problemas de saúde mental) $O R$ (transtorno mental) $O R$ (depressão) AND (quilombola) OR (quilombo).

As buscas foram executadas de forma independente por dois juízes, nas bases de dados indexadas na Lilacs, Medline e SciELO, em março de 2018. Durante esse processo, não foi delimitado o ano de publicação dos estudos, a fim de construir uma revisão abrangente. A seleção inicial resultou em 183 artigos, sendo 66 na Lilacs, 36 na Medline e 81 na SciELO, conforme indicado na Figura 1. Na base Lilacs, foram identificados 12 estudos duplicados, e na SciELO, 34; na Medline, não se encontrou duplicação e, entre as bases, o número de artigos duplicados somou 40.

Os resumos desses estudos foram analisados de acordo com o seguinte critério de inclusão: artigos empíricos realizados no Brasil que abordam os fatores inerentes ao sofrimento mental nas comunidades quilombolas. Um total de 80 artigos não atendeu ao critério de inclusão. Os 17 artigos restantes foram analisados de acordo com os seguintes critérios de exclusão: a) teses e dissertações; b) resumos de eventos e capítulos de livros. Nove foram excluídos por se enquadrarem nesses critérios. Dados os critérios de inclusão e exclusão, oito artigos foram identificados como relevantes.

Os pesquisadores analisaram, de forma independente, os artigos relevantes e emitiram parecer sobre a inclusão ou não desses na revisão. Depois do processo de seleção dos estudos e definição daqueles que seriam incluídos na revisão, os pesquisadores fizeram a tabulação dos dados extraídos em uma planilha que incluía título e autores, revista, objetivo, métodos, principais resultados e conclusão do estudo. Essas informações foram tabeladas buscando-se padrões que pudessem organizar os estudos. 
Figura 1 - Fluxograma do processo de obtenção de artigos

(Saúde mental or problemas de saúde mental or transtorno mental or depressão) AND (quilombola or quilombo)

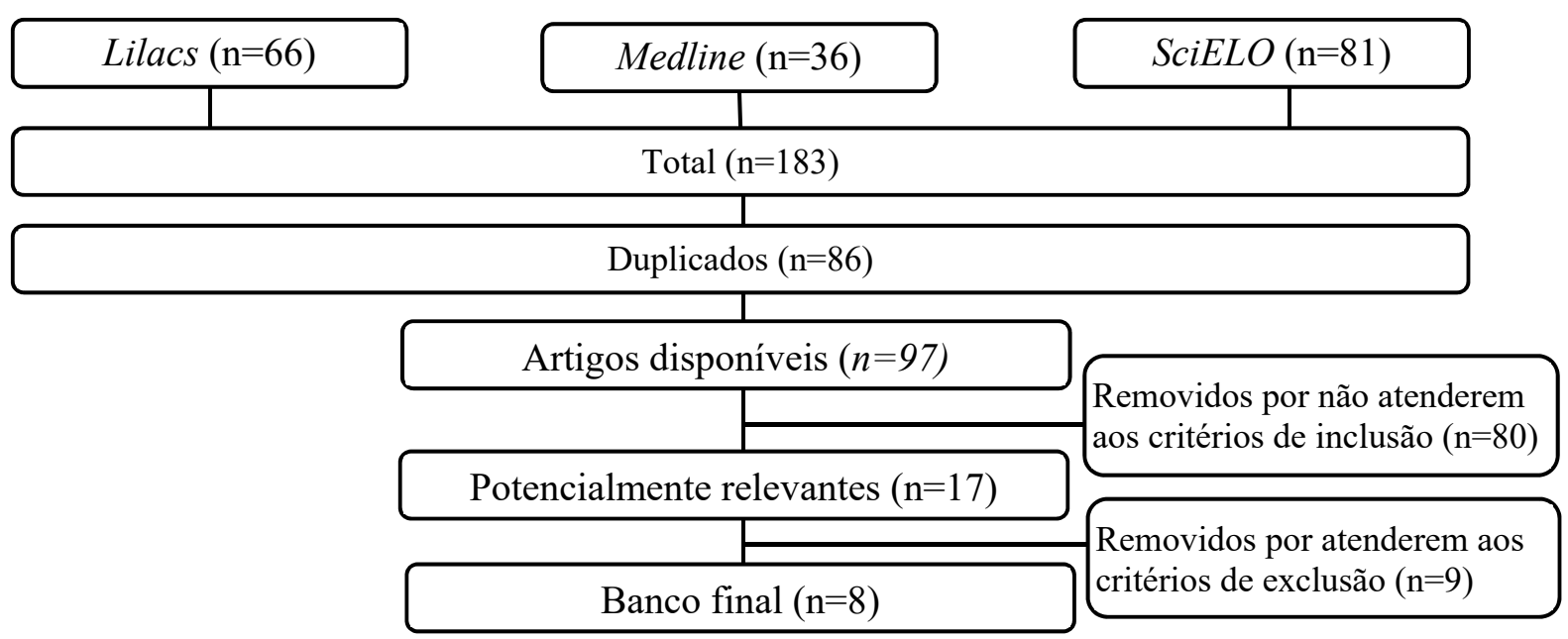

Fonte: os autores.

\section{RESULTADOS E DISCUSSÃO}

Com a finalidade de apresentar as informações dos estudos, apresenta-se no Quadro 1, de maneira sintética, a classificação das pesquisas conforme título, periódico, autoria e ano de publicação, objetivo, método, principais resultados e conclusão. A apresentação dos resultados e da discussão está dividida nas seguintes seções: caracterização geral dos artigos; categorização das amostras; indicadores sociais e econômicos das comunidades quilombolas; e marcadores sociais e fatores associados à condição de saúde mental nas comunidades.

\subsection{Caracterização dos artigos}

Os artigos foram publicados entre 2007 e 2016, sendo sete deles publicados entre 2014 e 2016. Quanto à área de publicação, a maioria foi publicada em periódicos da área da saúde $(n=6)$; um nas ciências sociais e um foi publicado em periódico de psicologia. Houve predominância de estudos realizados na região Nordeste $(n=7)$; apenas um foi realizado no Sudeste. Esse resultado pode ser explicado pela maior concentração de comunidades quilombolas naquela região. 


\begin{tabular}{|c|c|c|c|}
\hline & 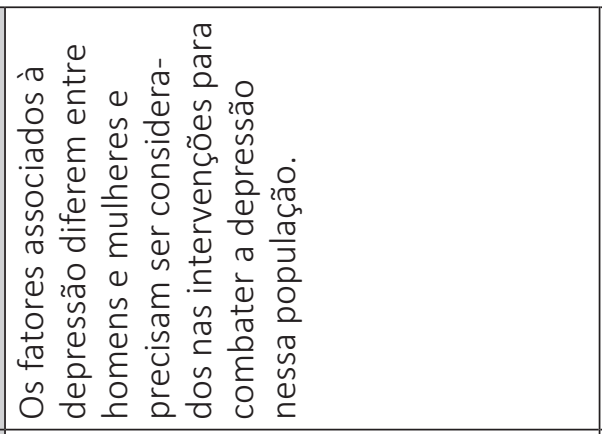 & 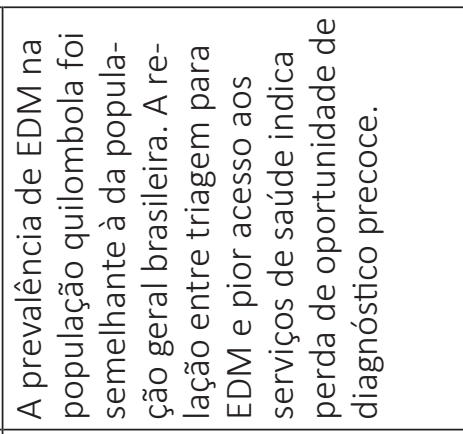 & 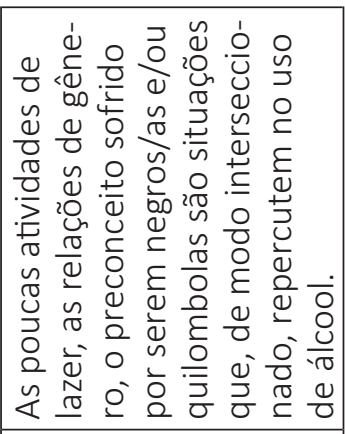 \\
\hline 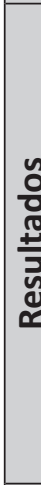 & 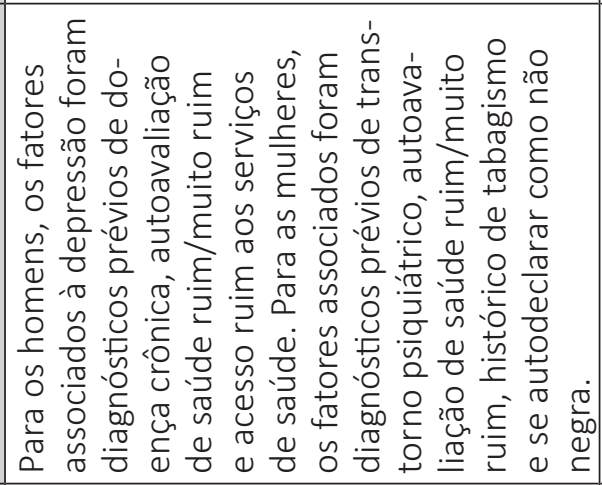 & 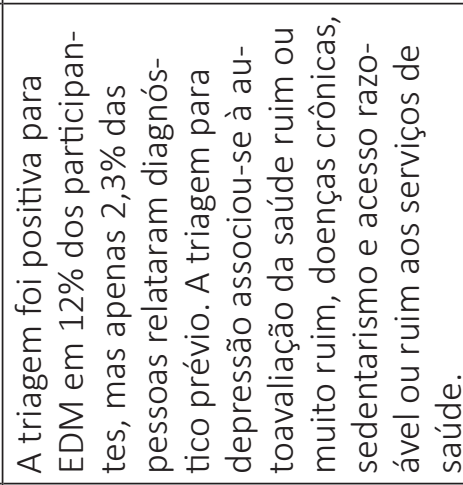 & 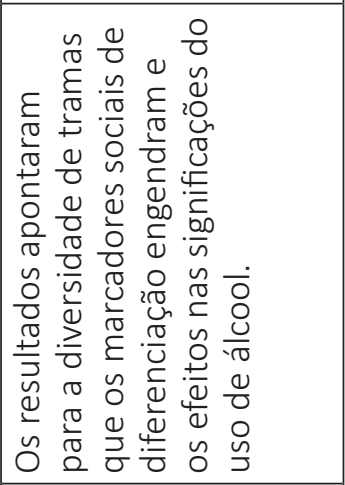 \\
\hline 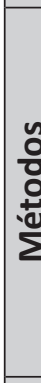 & 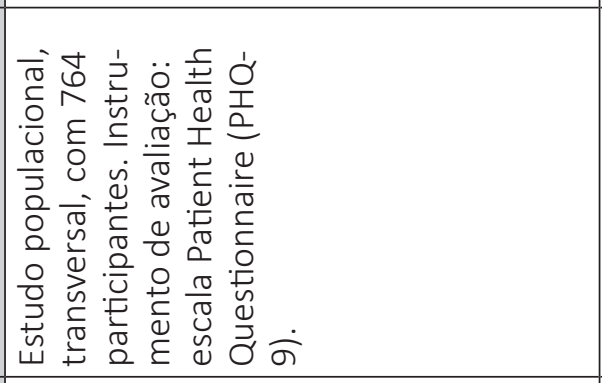 & 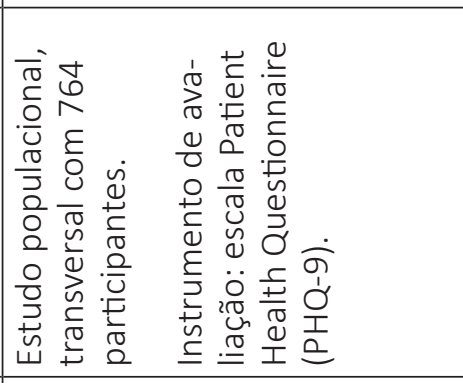 & 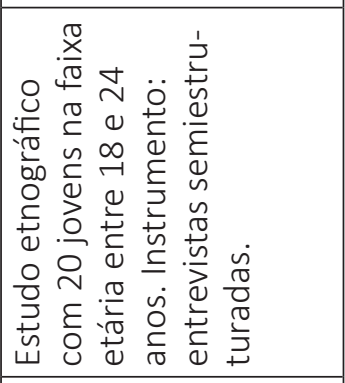 \\
\hline ?ִ & 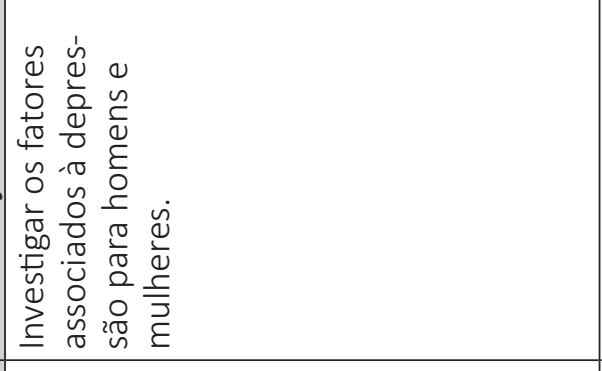 & 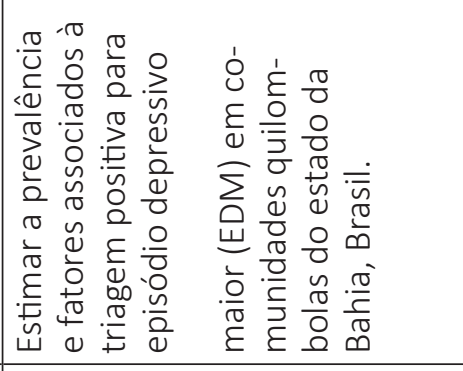 & 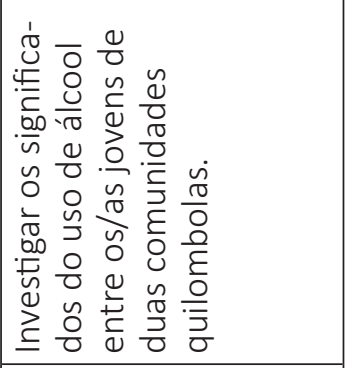 \\
\hline 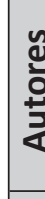 & 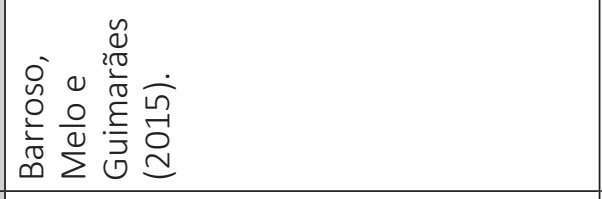 & 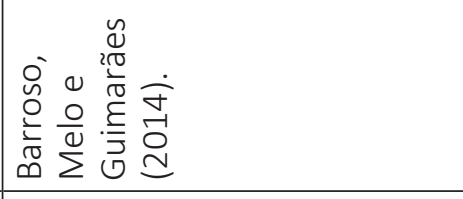 & 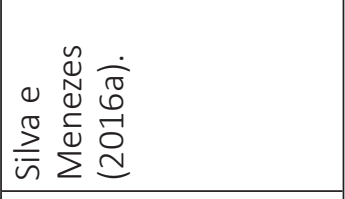 \\
\hline$\frac{\pi}{5}$ & 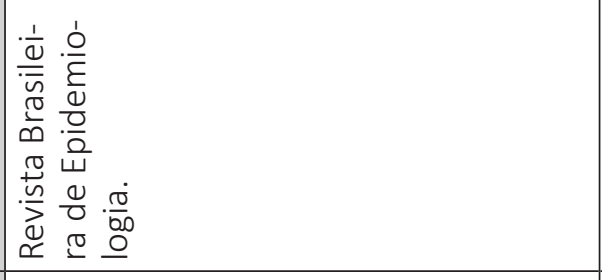 & 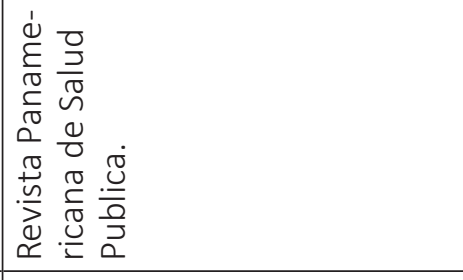 & 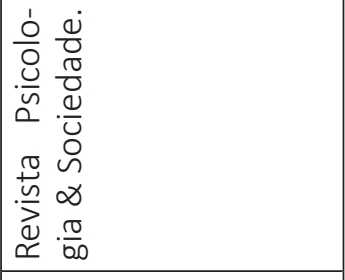 \\
\hline$\frac{0}{3}$ & 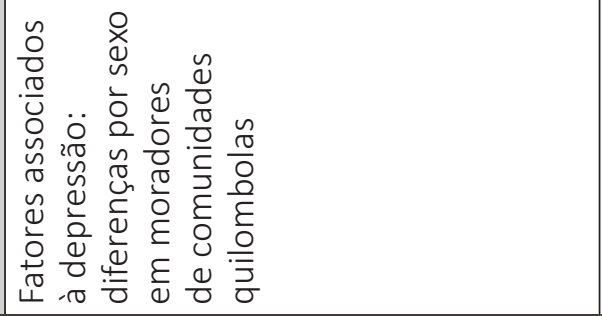 & 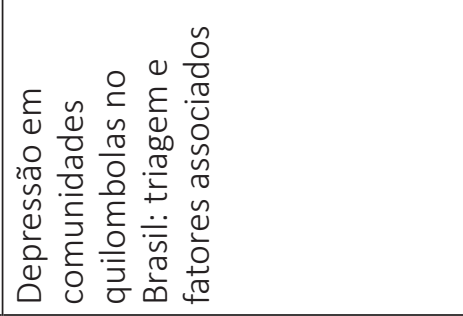 & 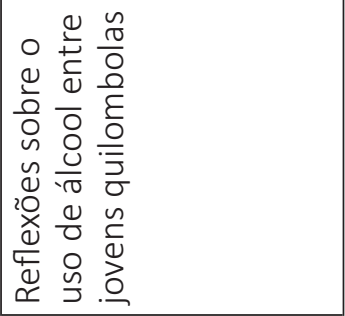 \\
\hline
\end{tabular}




\begin{tabular}{|c|c|c|c|}
\hline & 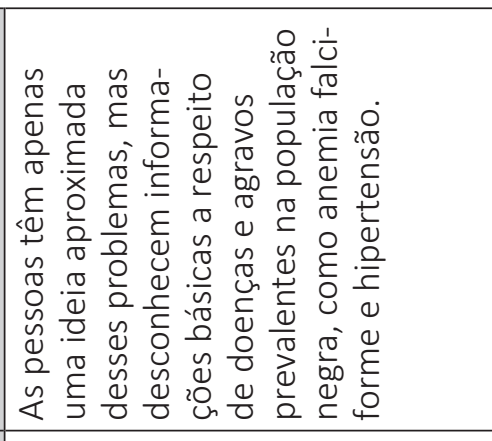 & 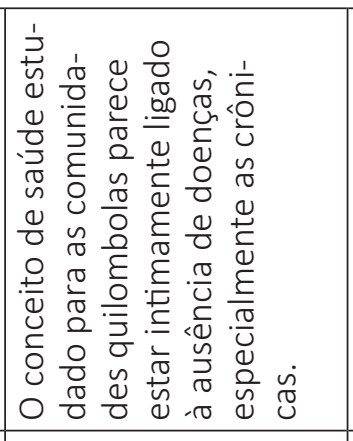 & 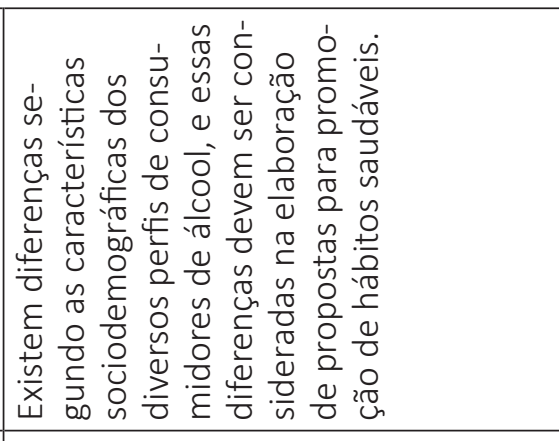 \\
\hline 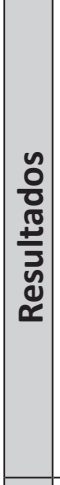 & 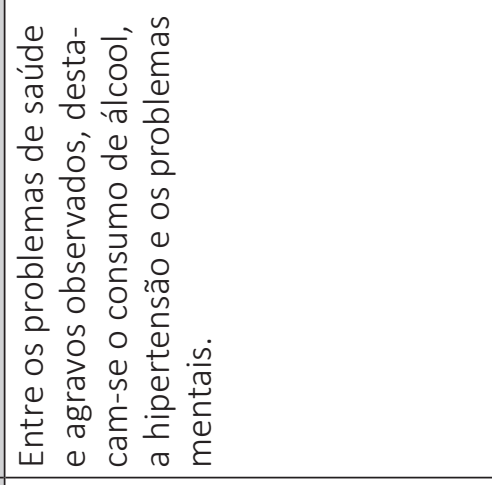 & 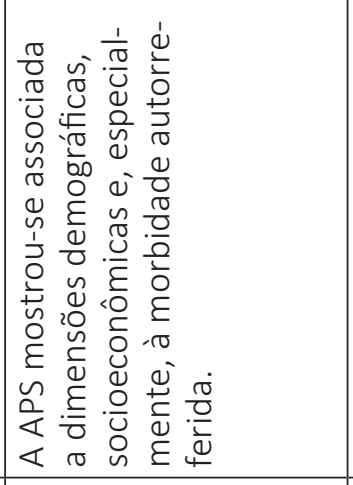 & 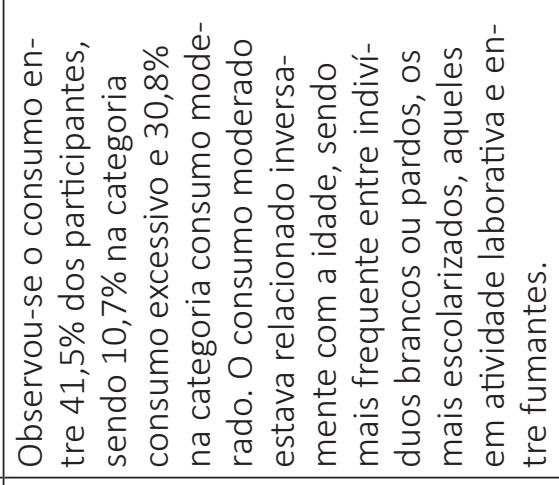 \\
\hline 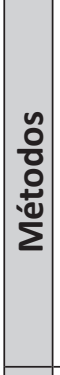 & 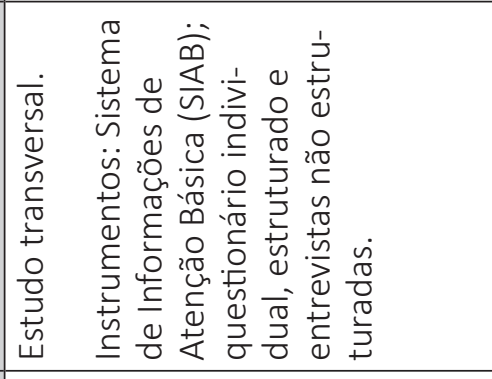 & 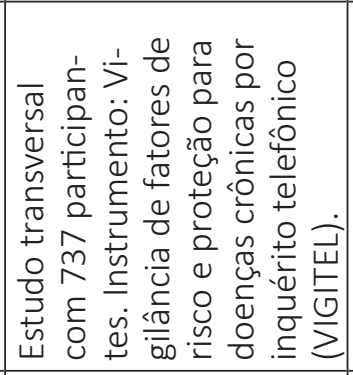 & 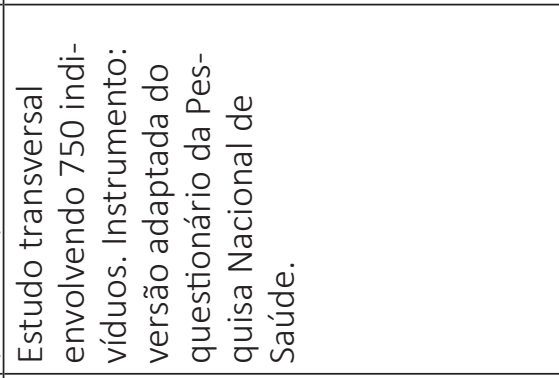 \\
\hline 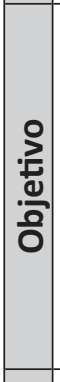 & 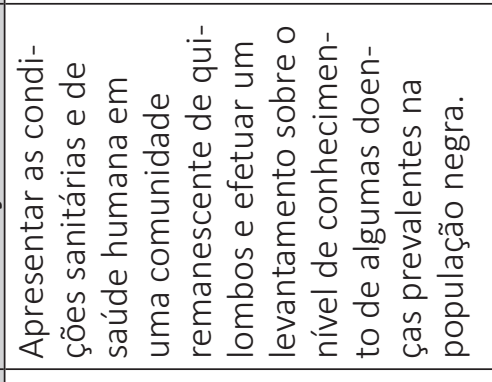 & 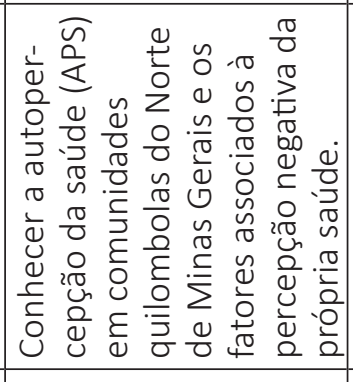 & 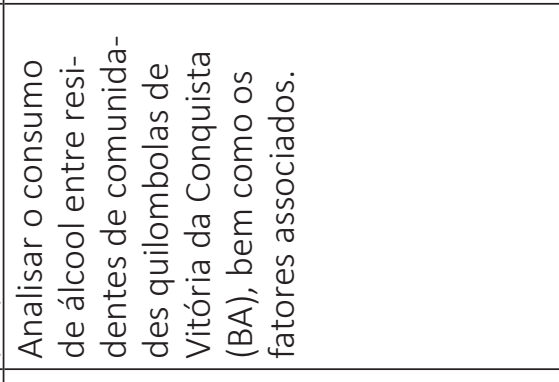 \\
\hline 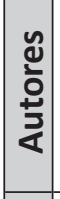 & 离 & 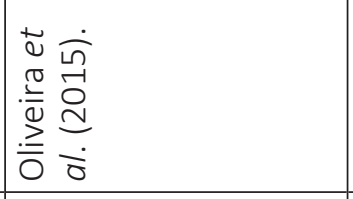 & 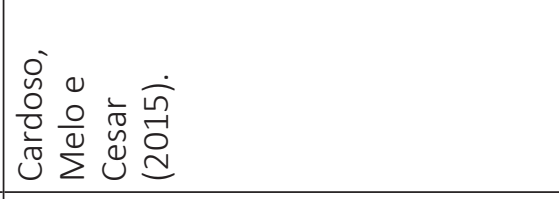 \\
\hline 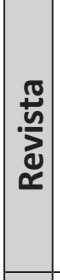 & 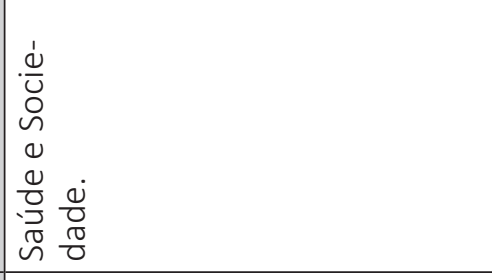 & 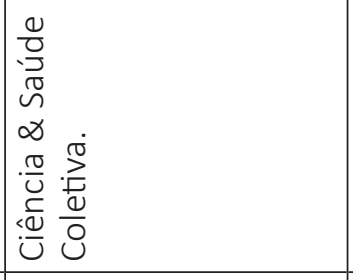 & 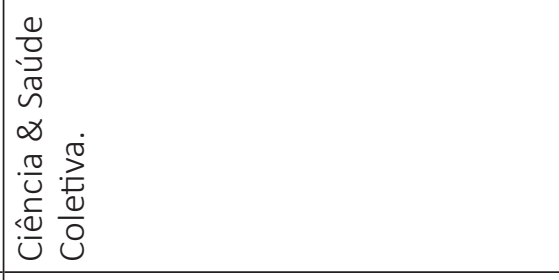 \\
\hline $\begin{array}{l}\frac{0}{3} \\
\stackrel{*}{*}=\end{array}$ & 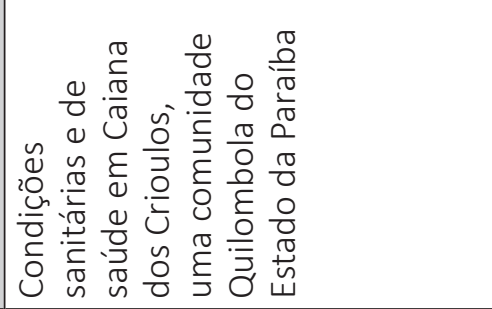 & 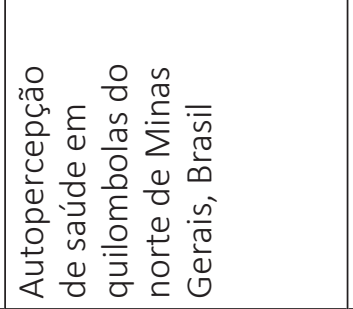 & 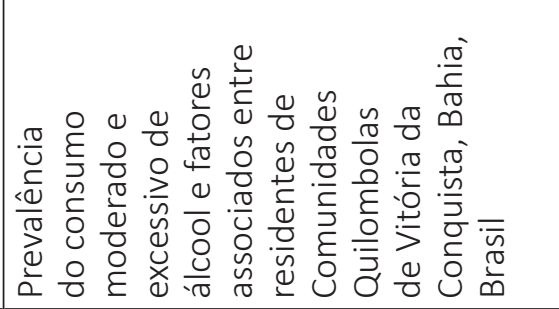 \\
\hline
\end{tabular}




\begin{tabular}{|c|c|}
\hline 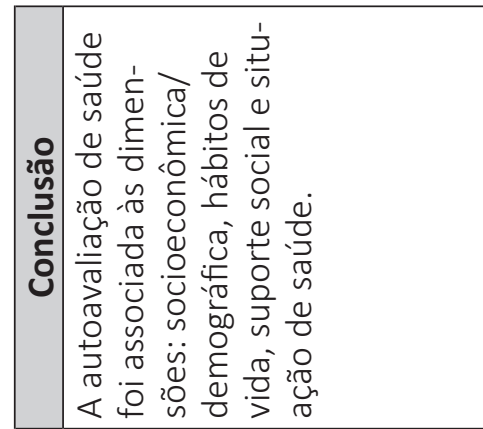 & 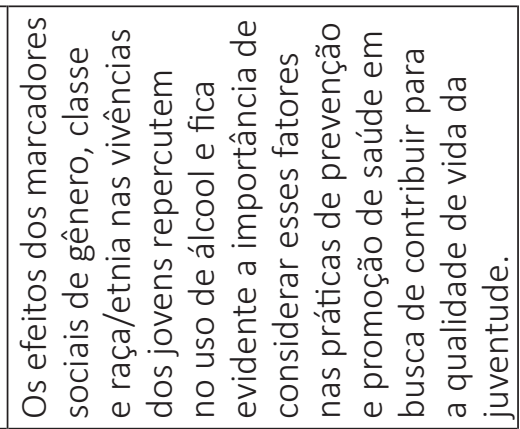 \\
\hline 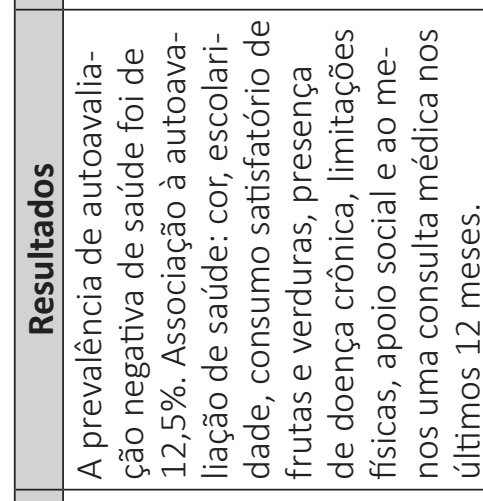 & 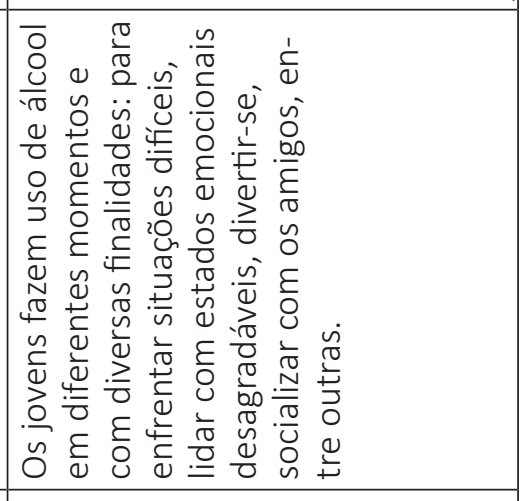 \\
\hline 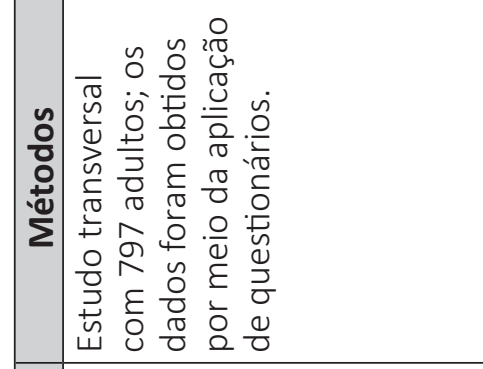 & 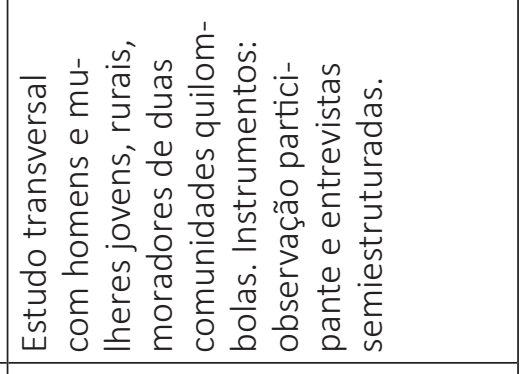 \\
\hline 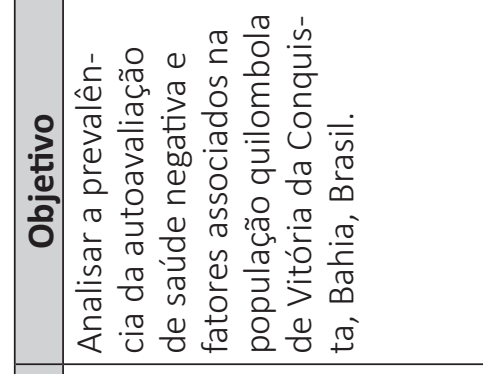 & 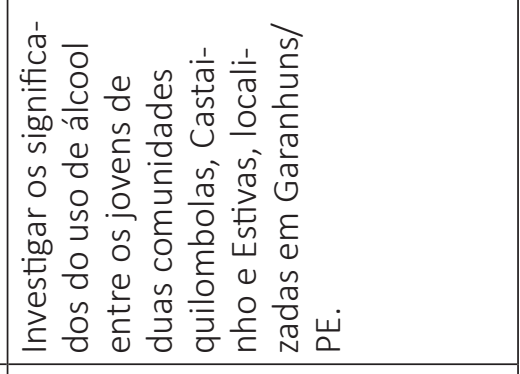 \\
\hline 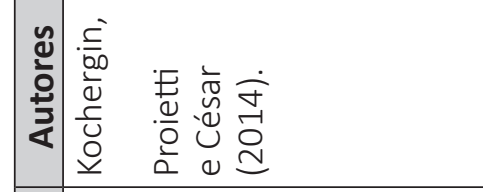 & 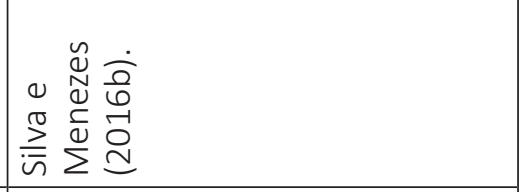 \\
\hline 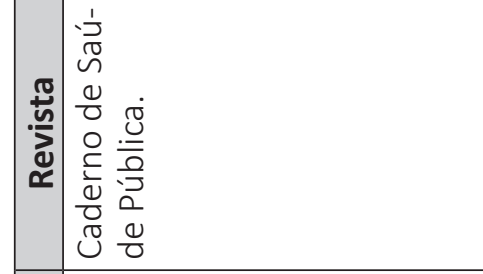 & 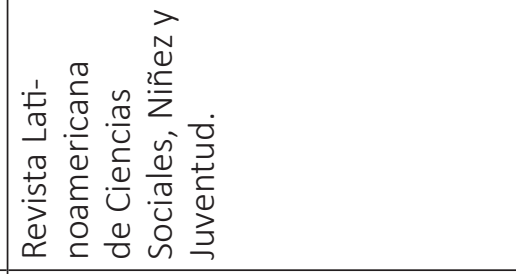 \\
\hline 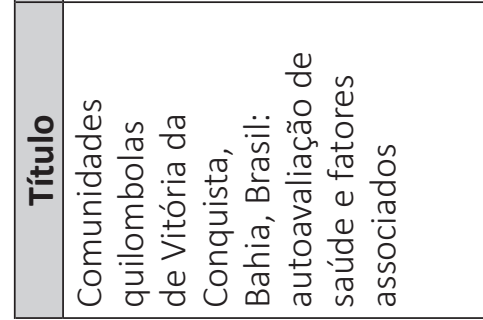 & 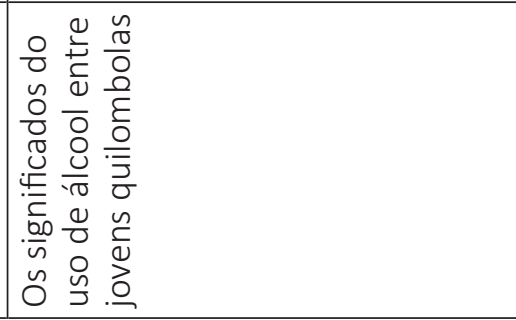 \\
\hline
\end{tabular}


Em relação aos objetivos dos estudos, três artigos buscaram investigar o consumo de álcool nas comunidades quilombolas (CARDOSO; MELO; CESAR, 2015; SILVA; MENEZES, 2016a; 2016b); destes, dois foram realizados com a mesma população. Outros três objetivaram analisar as condições e a autopercepção de saúde entre os quilombolas (SILVA, 2007; KOCHERGIN; PROIETTI; CÉSAR, 2014; OLIVEIRA et al., 2015) e dois avaliaram a depressão e os fatores associados (BARROSO; MELO; GUIMARÃES, 2014; 2015); estes últimos relatam resultados de uma mesma amostragem.

Quanto à metodologia utilizada, sete pesquisas adotaram como delineamento o estudo transversal (BARROSO; MELO; GUIMARÃES, 2014; 2015; SILVA, 2007; OLIVEIRA et al., 2015; CARDOSO; MELO; CESAR, 2015; KOCHERGIN; PROIETTI; CÉSAR, 2014; SILVA; MENEZES, 2016b). De acordo com Rouquayrol (2013), as pesquisas transversais de delineamento amostral, por ser um estudo epidemiológico em que o fator e o efeito são observados em um mesmo momento histórico, atualmente, têm sido as mais empregadas na área de saúde pública.

A maioria dos estudos (cinco) utilizou o método quantitativo como abordagem metodológica. Esse número pode ser explicado pela área à qual está vinculada a maioria dos periódicos em que esses artigos foram publicados. Uma pesquisa utilizou-se do método misto, e duas pesquisas, do método qualitativo. As pesquisas qualitativas tiveram como instrumento de coleta de dados a entrevista semiestruturada e observação; os estudos quantitativos utilizaram questionários, inquéritos e versão adaptada do questionário da Pesquisa Nacional de Saúde Patient Health Questionnaire (PHQ-9). O estudo de método misto utilizou-se do formulário produzido pelo Ministério da Saúde, Sistema de Informações de Atenção Básica (SIAB), questionário e entrevista não estruturada.

\subsection{Caracterização das amostras}

Em relação ao sexo dos participantes, quatro pesquisas apontaram predominância do sexo feminino: 64,2\% ( $n=485$ ) (OLIVEIRA et al., 2015); 53,5\% ( $n=401$ ) (CARDOSO; MELO; CESAR, 2015); 53,5\% ( $n=409$ ) (BARROSO; MELO; GUIMARÃES, 2015); e 53,3\% ( $n=398)$ (KOCHERGIN; PROIETTI; CÉSAR, 2014). Apenas no artigo de Silva (2007), o sexo masculino apresentou-se discretamente superior a o feminino, com 50,19\% ( $n=282$ ). Dois artigos não trouxeram informação precisa sobre o percentual de cada sexo.

A idade entre todos os estudos compreendeu a faixa etária entre 18 e 40 anos. Dois estudos foram realizados exclusivamente com jovens de idades entre 18 e 24 anos (SILVA; MENEZES, 2016a; 2016b). Em relação à cor da pele autorreferida pelos entrevistados, os estudos de Kochergin, Proietti e César (2014) obtiveram como resposta 39,0\% ( $n=291$ ) preto e 44,0\% $(n=330)$ pardo. No artigo de Cardoso, Melo e Cesar (2015), a cor preta foi autodeclarada por 41,6\% ( $n=312)$, e a parda, 44,0\% ( $n=330)$. No estudo realizado por Barroso, Melo e Guimarães (2014), o qual apresentava apenas duas opções de respostas, "negra" e "não negra", 84,03\% $(n=642)$ dos entrevistados responderam como afirmativa a cor negra. Por outro lado, no estudo de Silva (2007), no qual havia outras opções de respostas, como morena, mulata e parda, apenas $39,19 \%$ se declararam de cor negra.

Com base na contribuição dos estudos analisados nesta revisão, duas temáticas foram elaboradas para a discussão dos resultados, visando à realização de uma discussão organizada e relevante: os indicadores sociais e econômicos das comunidades quilombolas; marcadores sociais e fatores associados à saúde mental nas comunidades. 


\subsection{Indicadores sociais e econômicos das comunidades quilombolas}

Compõem este tema os resultados das pesquisas que caracterizavam o contexto em que se encontravam os participantes. Como mencionado anteriormente, grande parte dos estudos foi realizada na região Nordeste do País. Em linhas gerais, os participantes dos estudos analisados apresentaram baixo nível de escolaridade. No estudo de Kochergin, Proietti e César (2014), 32,4\% ( $n=241)$ dos entrevistados revelaram não ter frequentado a escola, e 28,0\% ( $n=208)$ frequentaram entre um e três anos; esses mesmos números se assemelham aos resultados encontrados por Cardoso, Melo e Cesar (2015), os quais mostraram que a média de escolaridade dos participantes foi de três anos de estudos, sendo que mais de 95\% dos indivíduos não completaram o ensino médio. No artigo de Barroso, Melo e Guimarães (2015), 34,9\% ( $n=124)$ dos homens e 32,3\% $(n=132)$ das mulheres não frequentaram a escola. A escolaridade no artigo de Oliveira et al. (2015) foi de 15,7\% ( $n=119$ ) para aqueles que nunca frequentaram a escola e de 44,4\% ( $n=336)$ para os que frequentaram entre um e quatro anos. Silva (2007), ao analisar a frequência escolar dos participantes de 0 a 18 anos, verificou que 65,52\% (n=44) estavam fora da sala de aula. Dois estudos não apresentaram a escolaridade dos participantes (SILVA; MENEZES, 2016a; 2016b).

Esses dados corroboram outros estudos que apontam para o baixo nível de escolaridade nas comunidades quilombolas em várias regiões do país, com um índice significativo de adultos que não sabem ler e nem assinar o próprio nome (PINHO et al., 2015; SANTOS; FÉLIX; MORAIS, 2012; MARQUES et al., 2014). O baixo índice de escolaridade nas comunidades quilombolas, sobretudo na população adulta, pode contribuir para a manutenção da pobreza e subdesenvolvimento dessas comunidades (PINHO et al., 2015), ampliando a desigualdade socioeconômica entre os grupos que se declaram pretos ou pardos e os brancos.

Essa desigualdade social pode ser observada no exemplo citado pelo IBGE ao analisar a ocupação por atividade econômica e nível de instrução da população brasileira entre os anos de 2012 e 2016. Os dados revelaram que, entre os trabalhadores pretos ou pardos, 34,7\% não tinham instrução ou tinham ensino fundamental incompleto, percentual que era de $21,2 \%$ para os trabalhadores brancos. Além disso, entre os trabalhadores pretos ou pardos, apenas $11,0 \%$ possuíam nível superior, enquanto entre trabalhadores brancos com o mesmo nível de instrução esse número é duas vezes maior, chegando a 26,4\% (IBGE, 2017).

Nesta revisão, ao analisar a ocupação dos participantes, pode-se observar que o número de pessoas desocupadas apontadas no estudo de Cardoso, Melo e Cesar (2015) totalizou 48,8\% $(n=366)$ da amostra investigada. Semelhante a esse resultado, no artigo de Barroso, Melo e Guimarães (2014), o número de participantes que declararam não estar trabalhando foi de 50,4\% $(n=385)$. O trabalho informal, dedicado à pequena agricultura, foi apontado como a ocupação de $98,24 \%$ dos homens respondentes da pesquisa de Silva (2007). Não possuir nenhuma fonte de renda foi declarado por 28,3\% ( $n=208)$ dos participantes do estudo de Kochergin, Proietti e César (2014). O crescimento da taxa de desocupação nos últimos anos tem ocorrido em todos os níveis de instrução e grupos populacionais, no entanto as taxas de desocupação da população preta ou parda foram superiores às da população branca em todos os níveis de instrução, sendo registrados, em 2016, os índices de 13,2\% e 9,1\%, respectivamente (IBGE, 2017).

Todas as pesquisas foram realizadas em comunidades quilombolas da área rural, fator este que aumenta o grau de exposição dessa população à desvantagem social ao se perpetuar o acesso desigual aos recursos materiais e de saúde pública oferecidos nos centros urbanos. A 
localização geográfico-espacial das comunidades quilombolas rurais, sobretudo das regiões Norte e Nordeste, que têm um acesso limitado aos serviços de atenção primária, se comparadas às residentes nas regiões Centro-Oeste, Sudeste e Sul, é um dos fatores que influenciam fortemente as desigualdades no acesso e utilização dos serviços de saúde (PEREIRA; SILVA; SANTOS, 2015).

A renda familiar foi investigada por apenas quatro estudos, os quais revelaram que a maioria dos entrevistados possuía renda menor que um salário mínimo (BARROSO; MELO; GUIMARÃES, 2014; 2015; KOCHERGIN; PROIETTI; CÉSAR, 2014; OLIVEIRA et al., 2015). Tais resultados colocam essas comunidades entre as populações de classe mais baixa do Brasil, chamadas Classe E. Contudo, Ferreira e Latorre (2012) postulam que, embora a maioria dos indicadores sociais utilizados nos estudos epidemiológicos esteja pautada na quantidade de renda e nos bens do indivíduo com base em dados estatísticos, a compreensão do fenômeno da desigualdade social necessita do entendimento da sua gênese e a sua reprodução no tempo. Vale lembrar que, no Brasil pós-abolição, a ideologia do branqueamento posta em prática com o incentivo dado aos imigrantes europeus suprimiu as oportunidades de trabalho para a população negra, sendo estas reservadas ao segmento branco, restando aos negros posições sociais inferiores ou rejeitadas pelos brancos, consequentemente, com remunerações menores (SANTOS, 2015).

Nesse sentido, os indicadores socioeconômicos apresentados nesta revisão corroboram resultados de outros estudos, os quais apontam as comunidades remanescentes de quilombolas como um grupo populacional que sofre com as iniquidades sociais, as quais afetam predominantemente as populações negras, pobres, rurais, com baixa escolaridade, sem acesso adequado a serviços de saúde e sem saneamento ambiental (MELO; SILVA, 2015; SANTOS; FÉLIX; MORAIS, 2012; PINHO et al., 2015; MONEGO et al., 2010).

\subsection{Marcadores sociais e fatores associados à condição de saúde mental nas comunidades}

Nessa categoria de análise, discutem-se os marcadores sociais como fatores de vulnerabilidade social que influenciam direta ou indiretamente nas condições de saúde mental da população estudada. Destarte, busca-se analisar o consumo de bebidas alcoólicas e a presença de outros transtornos mentais presentes nas investigações desta revisão.

A presença de consumo abusivo de álcool foi apontada em todos os oito estudos analisados. Em cinco deles, a variável consumo de bebidas alcoólicas foi investigada como categoria sociodemográfica (OLIVEIRA et al., 2015; BARROSO; MELO; GUIMARÃES, 2014; 2015; SILVA, 2007; KOCHERGIN; PROIETTI; CÉSAR, 2014). Nas outras pesquisas, o consumo de álcool foi o objetivo do estudo.

Em seus estudos, Silva e Menezes (2016a; 2016b) buscaram investigar os significados do uso de álcool entre jovens de duas comunidades quilombolas. Os resultados encontrados nesses dois artigos mostraram que o uso de álcool entre os jovens entrevistados estava associado a diversos fatores, como o enfrentamento de situações difíceis; o preconceito por serem negros e/ou quilombolas; para lidar com estados emocionais diversos; suprir a falta de atividades de lazer ou, ainda, para se divertirem e socializarem com os amigos. Esses dados se assemelham aos achados de Soares et al. (2017) sobre os motivos que levaram jovens e adultos a ingerirem bebidas alcoólicas. Na referida pesquisa, destacaram-se respostas como: para interagir com colegas, distrair-se de alguma situação/problema, aliviar tensão/estresse; beber por lazer; ter expectativa em algo positivo; e ter aumento de confiança em situações com outras pessoas. 
Nos estudos desta revisão, o consumo de bebidas alcoólicas ganha significados distintos quando analisado sob as perspectivas das relações de gênero. Para os homens, o ato de consumir a bebida é visto como afirmação da sua masculinidade e como algo que faz parte da sua cultura. É compreendido também como compensação pelo trabalho perigoso que executam, além de relaxar e distrair. Já para as mulheres da comunidade, beber ajuda a lidar com os sentimentos como raiva, paixão etc.; significa esquecer os problemas, inibir sensações negativas, ou seja, o álcool é visto como fonte de liberdade (SILVA; MENEZES, 2016a; 2016b).

Esses estudos revelam que o consumo de bebidas alcoólicas como marcador social para esses jovens é uma prática comum, "normal", marcada por padrões culturais construídos socialmente por meio da exclusão. As dificuldades de acesso às oportunidades oferecidas nos centros urbanos e a ausência de políticas públicas na criação e efetivação de ações de viabilização de um ensino de qualidade, investimentos em áreas de lazer, esporte e entretenimento nessas comunidades parecem provocar nesses jovens baixas perspectivas sobre o futuro, e, consequentemente, a bebida se torna um refúgio.

Ainda nessa linha de estudo, a investigação de Cardoso, Melo e Cesar (2015) teve por objetivo analisar o consumo moderado e excessivo de álcool e fatores associados entre residentes de uma comunidade quilombola. Nessa pesquisa, os resultados mostraram que $41,5 \%$ ( $n=311)$ consumiam bebidas alcoólicas; destes, 10,7\% ( $n=80)$ faziam uso excessivo, e 30,8\%, $(n=231)$ uso moderado, sendo a maioria do sexo masculino e com menos de um ano de estudo, na faixa etária de 25 a 34 anos. Na referida pesquisa, o consumo excessivo de bebidas alcoólicas estava associado a viver sem companheiro(a); os fumantes mostraram maior propensão ao uso de álcool, especialmente excessivo, e foram encontradas prevalências maiores observadas entre cor da pele preta. Observa-se que esses dados superam o índice nacional divulgado pela Pesquisa Nacional de Saúde (PNS), em que a proporção de pessoas de 18 anos de idade ou mais que consumiam bebida alcoólica uma vez ou mais por semana era de 24,0\% (IBGE, 2013).

Outros transtornos mentais foram identificados como fatores associados à condição de saúde da população investigada. No estudo de Kochergin, Proietti e César (2014), 13,5\% dos entrevistados afirmaram se sentir deprimidos. Dados semelhantes $(13,5 \%)$ foram encontrados no estudo de Oliveira et al. (2015). Já nos estudos de Cardoso, Melo e Cesar (2015), o mesmo transtorno foi relatado por $19,1 \%$ dos respondentes. Em tais estudos, observou-se um número superior de indivíduos com relato de depressão em relação ao encontrado nacionalmente, que é de 7,6\% na população adulta, segundo a Pesquisa Nacional de Saúde divulgada pelo IBGE (2013).

Nesta revisão, dois estudos foram selecionados, a saber: Barroso, Melo e Guimarães (2014; 2015), os quais investigaram a depressão nas comunidades quilombolas. O primeiro analisou a prevalência de fatores associados à triagem positiva para Episódio Depressivo Maior (EDM) nas comunidades. Os resultados do estudo sobre EDM mostraram que a triagem foi positiva para $12 \%$ dos participantes e se associou à autoavaliação da saúde ruim ou muito ruim, doenças crônicas, sedentarismo e acesso razoável ou ruim aos serviços de saúde.

No segundo estudo, Barroso, Melo e Guimarães (2015) investigaram os fatores associados à depressão para homens e mulheres. Nessa pesquisa, os fatores associados à depressão para os homens foram diagnósticos prévios de doença crônica, autoavaliação de saúde ruim/muito ruim e acesso ruim aos serviços de saúde; e, para as mulheres que se autodeclararam como negras, foram o diagnóstico prévio de transtorno psiquiátrico, autoavaliação ruim/muito ruim e histórico de tabagismo. De forma geral, os resultados apontaram para diferenças por sexo, com 
prevalência de depressão maior para o sexo feminino. Além disso, outros marcadores sociais, como baixa escolaridade, condições de moradia, cor, desemprego, consumo de bebidas alcoólicas e renda familiar, associados à depressão, revelam que essas comunidades se encontram em situação de alta vulnerabilidade social.

Vale lembrar que o racismo no Brasil afeta diretamente a saúde mental da população não branca (preta e parda), parcela majoritária da população brasileira, ao lidar com ameaças à autoestima, desigualdades de oportunidades e com a violência, fatores que podem levar essa população ao sofrimento psíquico em formas e intensidades diversas (BRASIL, 2016).

\section{CONSIDERAÇÕES FINAIS}

No presente estudo, buscou-se, mediante uma revisão sistemática das publicações científicas nacionais, identificar estudos sobre saúde mental desenvolvidos nas populações quilombolas e se esses estudos consideram os determinantes sociais da saúde para compreender o processo de saúde e doença dessa população.

As pesquisas mostraram que o consumo abusivo de bebidas alcoólicas presente nas comunidades pode ser compreendido como uma prática cultural construída socialmente nessa população como forma de enfrentamento das desigualdades sociais vivenciadas, sobretudo, entre a população mais jovem (SILVA; MENEZES, 2016a; 2016b; CARDOSO; MELO; CESAR, 2015). Da mesma forma, os fatores associados aos sintomas depressivos encontrados nesta revisão sugerem a existência de uma relação entre as precárias condições de vida dessas pessoas e o seu sofrimento psíquico (BARROSO; MELO; GUIMARÃES, 2015). Contudo nenhum dos estudos teve por objetivo principal investigar as relações entre os indicadores sociais e a saúde mental dos entrevistados.

Outras limitações foram identificadas ao longo da elaboração do presente artigo, relacionadas principalmente à qualidade de alguns estudos analisados e seus resultados. Como observado na discussão anteriormente exposta, apenas cinco estudos tiveram como objetivo analisar transtornos mentais nas comunidades. Também deve ser considerada a limitação relacionada à falta de estudos sobre determinantes sociais da saúde mental nas comunidades quilombolas. Outra limitação está relacionada à inclusão apenas de artigos resultantes de pesquisas nacionais.

Outra lacuna a ser observada é a escassez de estudos sobre os determinantes sociais da saúde mental da comunidade quilombola publicados em periódicos de psicologia. É com base nessa constatação que se faz indispensável a realização de mais estudos que vão além da investigação epidemiológica dessa população, mas que também tenham como objeto a compreensão desses indicadores sociais que historicamente contribuíram para a manutenção das desigualdades em que se encontra esse grupo. Sugere-se, também, que a realização de estudos com outras comunidades rurais se faz necessária à compreensão da problemática vivida pelas comunidades quilombolas.

\section{REFERÊNCIAS}

ALVES, A. A. M.; RODRIGUES, N. F. R. Determinantes sociais e económicos da Saúde Mental. Revista Portuguesa de Saúde Pública, Lisboa, v. 28, n. 2, p. 127-31, 2010.

BARROSO, S. M.; MELO, A. P.; GUIMARÃES, M. D. C. Fatores associados à depressão: diferenças por sexo em moradores de comunidades quilombolas. Revista Brasileira de Epidemiologia, São Paulo, v. 18, n. 2, p. 503-14, 2015. 
BARROSO, S. M.; MELO, A. P.; GUIMARÃES, M. D. C. Depressão em comunidades quilombolas no Brasil: triagem e fatores associados. Revista Panamericana de Salud Pública, v. 35, n. 4, p. 256-63, 2014.

BEZERRA, V. M. et al. Inquérito de saúde em comunidades quilombolas de Vitória da Conquista, Bahia, Brasil (Projeto COMQUISTA): aspectos metodológicos e análise descritiva. Ciência \& Saúde Coletiva, Rio de Janeiro, v. 19, n. 6, p. 1835-47, 2014.

BOSI, M. L. M. et al. Determinantes sociais em saúde (mental): analisando uma experiência não governamental sob a ótica de atores implicados. Revista Brasileira de Epidemiologia, São Paulo, v. 17, supl. 2, p. 126-35, 2014.

BRASIL. Ministério da Saúde. Secretaria de Gestão Estratégica e Participativa. Departamento de Articulação Interfederativa. Temático Saúde da População Negra/Ministério da Saúde. Brasília, DF: Ministério da Saúde, 2016.

BRASIL. Ministério da Saúde. Portaria n. 992, de 13 de maio de 2009. Institui a Política Nacional de Saúde Integral da População Negra. Diário Oficial [da] República Federativa do Brasil, Brasília, DF, 14 maio 2009. Seção 1.

BUSS, P. M.; PELLEGRINI FILHO, A. A saúde e seus determinantes sociais. Physis: Revista de Saúde Coletiva, Rio de Janeiro, v. 17, n. 1, p. 77-93, 2007.

CAVALCANTE, I. M. S. Acesso e acessibilidade aos serviços de saúde em Três Quilombos da Amazônia Paraense: um olhar antropológico. 2011. Dissertação (Mestrado em Saúde, Sociedade e Endemias na Amazônia)- Universidade Federal do Pará, Belém, 2011.

CARDOSO, L. G. V.; MELO, A. P. S.; CESAR, C. C. Prevalência do consumo moderado e excessivo de álcool e fatores associados entre residentes de Comunidades Quilombolas de Vitória da Conquista, Bahia, Brasil. Ciência \& Saúde Coletiva, Rio de Janeiro, v. 20, n. 3, p. 809-20, 2015.

DIMENSTEIN, M. et al. Determinação social da saúde mental: contribuições à psicologia no cuidado territorial. Arquivos Brasileiros de Psicologia, Rio de Janeiro, v. 69, n. 2, p. 72-87, 2017.

FERREIRA, M. A. F.; LATORRE, M. R. D. O. Desigualdade social e os estudos epidemiológicos: uma reflexão. Ciência \& Saúde Coletiva, Rio de Janeiro, v. 17, n. 9, p. 2523-31, 2012.

FREITAS, D. A. et al. Saúde e comunidades quilombolas: uma revisão da literatura. Revista CEFAC, São Paulo, v. 13, n. 5, p. 937-43, set./out. 2011.

GAMA, C. A. P.; CAMPOS, R. T. O.; FERRER, A. L. Saúde mental e vulnerabilidade social: a direção do tratamento. Revista Latinoamericana de Psicopatologia Fundamental, São Paulo, v. 17, n. 1, p. 69-84, mar. 2014.

IBGE. Síntese dos Indicadores Sociais. Rio de Janeiro, 2017.

IBGE. Síntese dos Indicadores Sociais. Rio de Janeiro, 2013.

KOCHERGIN, C. N.; PROIETTI, F. A.; CÉSAR, C. C. Slave-descendent communities in Vitória da Conquista, Bahia State, Brazil: self-rated health and associated factors. Cadernos de Saúde Pública, Rio de Janeiro, v. 30, n. 7, p. 1487-501, 2014.

KRIEGER, N. A glossary for social epidemiology. Journal of Epidemiology \& Community Health, v. 55, n. 10, p. 693-700, 2001. 
MARQUES, A. S. et al. Atenção primária e saúde materno-infantil: a percepção de cuidadores em uma comunidade rural quilombola. Ciência \& Saúde Coletiva, Rio de Janeiro, v. 19, n. 2, p. 365-71, 2014.

MELO, M. F. T.; SILVA, H. P. Doenças crônicas e os determinantes sociais da saúde em comunidades quilombolas do Pará, Amazônia, Brasil. Revista da Associação Brasileira de Pesquisadores/as Negros/as (ABPN), Uberlândia, MG, v. 7, n. 16, p. 168-89, mar./jun. 2015.

MONEGO, E. T.; Peixoto, M. R. G. (In) segurança alimentar de comunidades quilombolas do Tocantins. Segurança Alimentar e Nutricional, Campinas, SP, v. 17, n. 1, p. 37-47, 2010.

MOHER, D. et al. Preferred reporting items for systematic reviews and meta-analyses: the PRISMA statement. Annals of Internal Medicine, v. 151, n. 4, p. 264-9, 2009.

OLIVEIRA, S. K. M. et al. Self-perceived health among 'quilombolas' in northern Minas Gerais, Brazil. Ciência \& Saúde Coletiva, Rio de Janeiro, v. 20, n. 9, p. 2879-90, 2015.

ORGANIZAÇÃO MUNDIAL DE SAÚDE (OMS). Relatório Mundial de Saúde. 2001. Disponível em: https:// www.who.int/whr/2001/en/whr01_po.pdf. Acesso em: 18 dez. 2019.

PEREIRA, L. L.; SILVA, H. P.; SANTOS, L. M. P. Projeto Mais Médicos para o Brasil: estudo de caso em comunidades quilombolas. Revista da Associação Brasileira de Pesquisadores/as Negros/as (ABPN), Uberlândia, MG, v. 7, n. 16, p. 28-51, 2015.

PINHO, L. et al. Condições de saúde de comunidade quilombola no norte de Minas Gerais. Revista de Pesquisa Cuidado É Fundamental On-line, Rio de Janeiro, v. 7, n. 1, p. 1847-55, jan./mar. 2015.

ROCHA, K. B. et al. Inequalities in mental health in the Spanish autonomous communities: a multilevel study. The Spanish Journal of Psychology, n. 18, p. 27-8, 2015.

ROUQUAYROL, M. Z. Epidemiologia e saúde. 5. ed. Rio de Janeiro: MEDSI, 2013.

SANTOS, M. F. S.; FÉLIX, L. B.; MORAIS, E. R. C. Representações sociais de juventude em uma comunidade quilombola do Agreste Pernambucano. Psico, Porto Alegre, v. 43, n. 4, p. 524-32, out./dez. 2012.

SANTOS, R. E. O marxismo e a questão racial no Brasil: reflexões introdutórias. Lutas Sociais, São Paulo, v. 19, n. 34, p. 100-13, jan./jun. 2015.

SILVA, J. A. Novaes da. Condições sanitárias e de saúde em Caiana dos Crioulos, uma comunidade Quilombola do Estado da Paraíba. Saúde e Sociedade, São Paulo, v. 16, n. 2, p. 111-24, maio/ago. 2007.

SILVA, R. A.; MENEZES, J. A. Reflexões sobre o uso de álcool entre jovens quilombolas. Psicologia \& Sociedade, Belo Horizonte, v. 28, n. 1, p. 84-93, 2016a.

SILVA, R. A.; MENEZES, J. A. Os significados do uso de álcool entre jovens quilombolas. Revista Latinoamericana de Ciencias Sociales, Niñez y Juventud, Manizales, Colombia, v. 14, n. 1, p. 493-504, 2016b.

SOARES, F. J. et al. Análise dos motivos dos jovens e adultos consumirem álcool. Id on Line Revista Multidisciplinar e de Psicologia, Jaboatão dos Guararapes, PE, v. 11, n. 35, p. 554-66, 2017.

SOUZA, D. O.; SILVA, S. E. V.; SILVA, N. O. Determinantes sociais da saúde: reflexões a partir das raízes da "questão social". Saúde e Sociedade, São Paulo, v. 22, n. 1, p. 44-56, 2013. 
VAREJÃO, V. A. et al. Fortalecimento da identidade e da autonomia da comunidade: um enfoque na saúde. In: SEMINÁRIO DO PROJETO INTEGRALIDADE: SABERES E PRÁTICAS NO COTIDIANO DAS INSTITUIÇÕES DE SAÚDE, 6., 2006. Rio de Janeiro. Anais [...]. Rio de Janeiro: ABRASCO, 2006. p. 119-26.

XAVIER, L. C. A visão da feminilidade sobre os cuidados em saúde dos quilombos contemporâneos. In: BATISTA, L. E.; WERNEK, J.; LOPES, F. (Org.). Saúde da população negra. Brasília, DF: ABPN - Associação Brasileira de Pesquisadores Negros, 2012. p. 204-21.

\section{Sobre os autores:}

Eraldo Carlos Batista: Doutor em Psicologia Social pela Pontifícia Universidade Católica do Rio Grande do Sul (PUCRS). Mestre em Psicologia pela Fundação Universidade Federal de Rondônia (UNIR). Especialista em Saúde Mental pela Universidade Católica Dom Bosco (UCDB). Bacharel em Psicologia. Professor do Departamento de Psicologia da Faculdade Católica de Rondônia (FCR). E-mail: eraldo.cb@hotmail.com, Orcid: http://orcid.org/0000-0002-7118-5888

Katia Bones Rocha: Doutora em Psicologia pela Universitat Autònoma de Barcelona (UAB), España. Mestre em Psicologia Social pela Pontifícia Universidade Católica do Rio Grande do Sul (PUCRS). Professora do Departamento de Psicologia da PUCRS. E-mail: katiabonesrocha@gmail.com, Orcid: http://orcid.org/0000-0001-7603-1709 